\title{
INGROWING TOE-NAILS
}

By C. E. KEMP

Head of the School of Chiropody, Chelsea Polytechnic, London

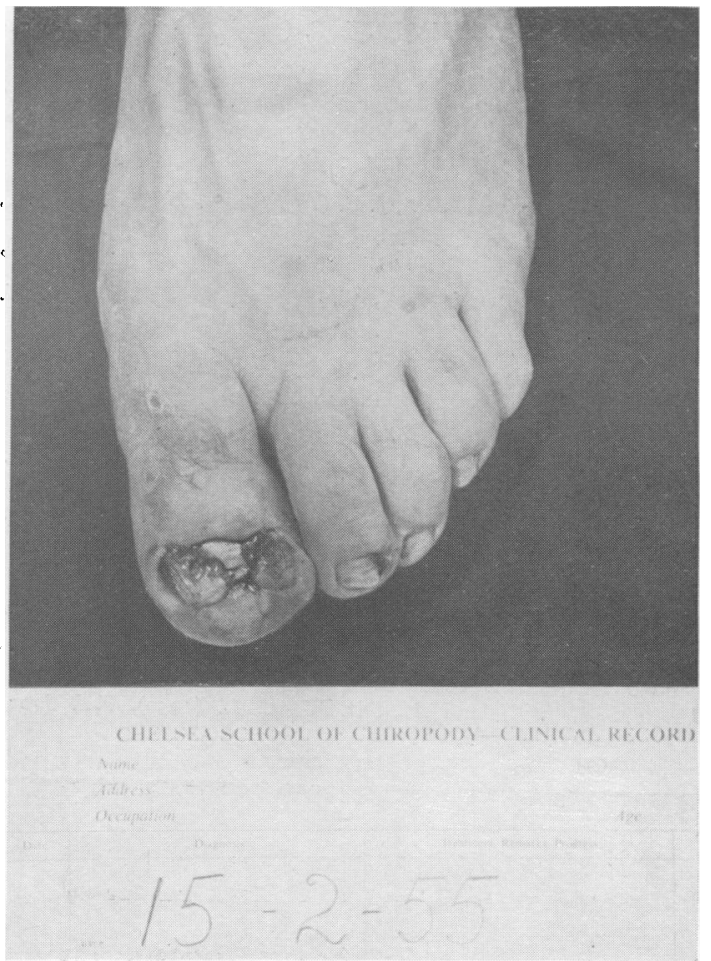

FIG. I.-Mr. R. C. Onychocryptosis complicated with massive granulation tissue. Condition present for 18 months. Note marked swelling of first toe.

The terms onychocryptosis, 'ingrowing nail' and ' ingrown nail' are used in various localities to indicate degrees of severity but for the purpose of this article they will be applied as synonyms to describe a condition where a portion of the side of the nail plate has actually penetrated the epidermis and dermis of the nail groove to produce a wound. The condition may or may not be further complicated by acute or chronic infection together with excessive granulation tissue partially or wholly obscuring the nail plate.

- A normal nail appears to arise from the deeply invaginated epidermal tissue of the dorsum of the

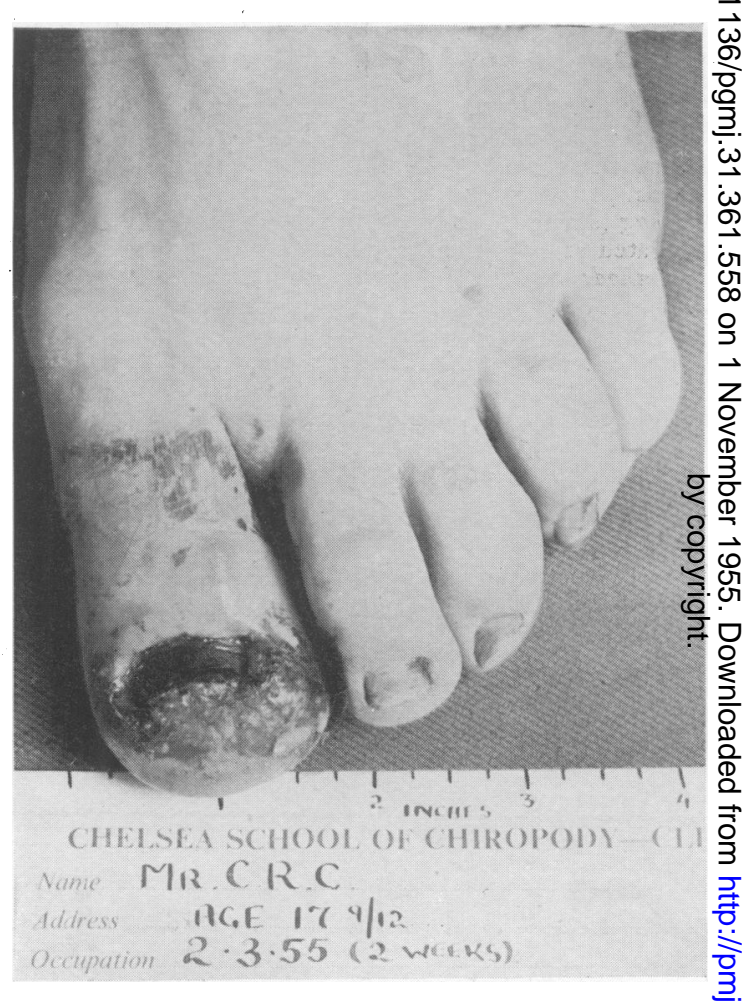

Fig. 2.-Mr. R. C. After two weeks' treatment. Note: Most of the nail plate now visible, some granulation tissue still present. The photograph shows 0 apparently dark colouring around the nail, this is actually the bright yellow stain of iron chloride and it does not obscure the field to the extent suggested by the illustration.

toe, the nail ' root' appearing to lie quite close to, $\widetilde{N}$ and protected by, the tuberosities at the base of $N$ the distal phalanx and roughly takes its transverse N contour from the shape of these structures. As $\sigma$ the tough, but flexible, nail plate moves smoothly and uneventfully forward with the relatively soft surface of the nail bed, the hardened front edge is $\stackrel{\Phi}{?}$ supported by the tough hyponychium and 7 although there may be considerable strains and ${ }^{\circ}$ stresses from both intrinsic and extrinsic factors, 


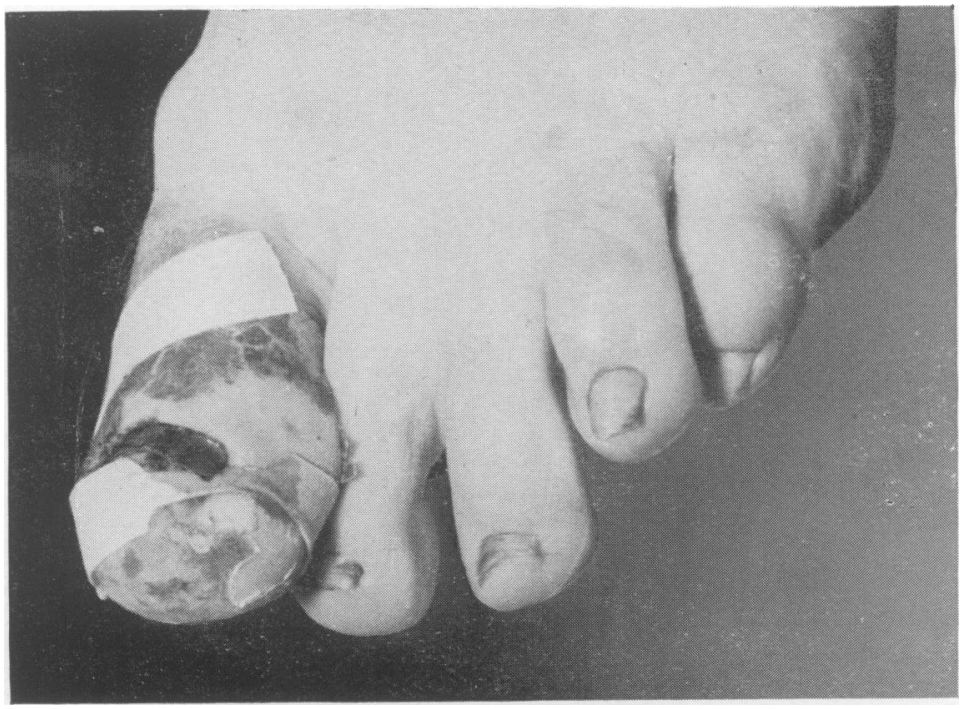

Fig. 3.-Mr. R. C. Five weeks. First sulcus strapping applied to lift nail edge and to depress the nail wall.

the nail plate offers surprising resistance to these deforming influences. If the nail plate is weakened by gross maceration through excessive perspiration, by incomplete development in severe general illness or by damage to the plate by fungoid infection, then very gross changes of the plate may be observed.

Where the patient has a general digital shape which is too large or too wide for the toe-cap of the shoes, or there is back pressure from the toe-box of a functionally short shoe, or from short or shrunken hosiery, then the direction of the forces normally acting on the nail plate may pass through an angle of almost $90^{\circ}$, i.e. either medially, distally or laterally due to the shoe resisting the forward thrust through the toe. The magnitude of these forces does not appear to have been measured, but inspection of the inside of the damaged toe-box will show that they must be considerable. In most of these cases investigation of the inside of the shoe shows a considerable depression in the hard toe-cap of the shoe where the big toe is being pressed into the leather of the shoe and causing still further obstruction to the nail plate. As the shape of the forepart of the shoe is beginning to show a considerable curvature at this point, the back thrust will not be along the centre line of the foot but will be at a considerable angle and the maximum compression area will often be on the lateral border of the nail plate where the small toes are crushed against the big toe which cannot move medially as it is securely held by the toe-box of the shoe. It is not always appreciated that the pressure by footwear mays force the soft tissues back against the nail and produce the same end result as the forward growth $\varnothing \mathrm{f}$ the nail plate against the obstructions in the nite groove. During the last 25 years there has bees. very notable reduction in the incidence of ingrown nail conditions requiring treatment by ours chiropodists and it is difficult to say whether thiso is due to improved foot fitting by the shoeo salesmen, better education by the health service or to better shapes of the forepart of shoes produced $\overrightarrow{\vec{F}}$ by the shoe manufacturers.

It is probably trite to state that if the entire? free edge of the nail plate rests on the hypo-0. nychium then an ingrown nail is impossible. The majority of cases occur because, for cosmetic 3 . reasons, patients try to produce oval toe-nailso similar to finger-nails and subsequently for. economic reasons they try to prevent holes ino hosiery by filing or cutting back the free edge so that the plate is no longer entirely supported by응 the hyponychium. The fibrous tissue binding the $>$ nail bed to the distal phalanx tends to pull the nailo down into the soft tissue so that it becomes increasingly difficult for the plate to make un-o interrupted forward progress. In order to relieve ${ }^{N}$ immediate pain the next stage is often a vigorous $N$ effort to remove the rough edge of nail plate witho scissors or nail nippers and initially this may beo successful, but the space in the groove is rapidly occupied by the soft tissues of the nail wall and any $\stackrel{D}{?}$ irregularities on the edge of the nail plate are still 0 more likely to be caught up than they would be in 


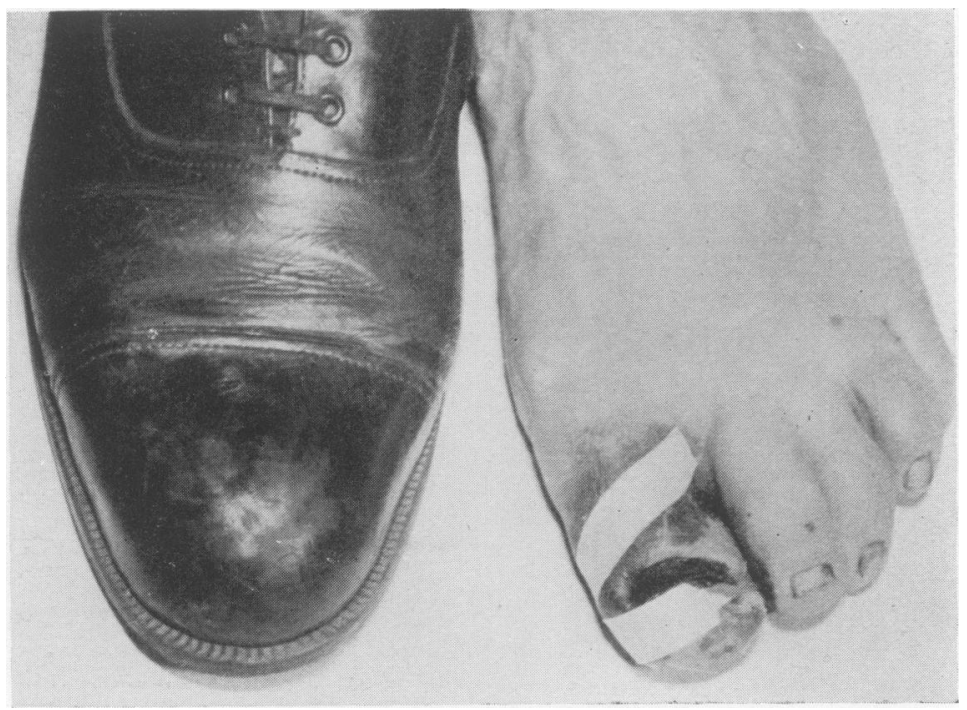

FIG. 4.-Mr. R. C. The immediate cause. A shoe which is much too narrow to accommodate the toes.

an untouched nail groove and the pain increases. The unsupported nail plate, subjected to side pressure against the shoe or to drag on either side in the 'peeptoe' shoe or sandal, now tends to become more tubular in shape and the medial and lateral edges may even touch or overlap and a cut made in this involuted nail plate will often produce a spear-like edge or splinter. (See illustration, page 562 ).

This projection is sufficiently pointed to penetrate the nail sulcus if the nail has been cut far back and the nail edge is thus situated deep to the soft nail walls. As the area becomes sensitive, still more of the corner of the nail plate is removed to obtain relief and this generally produces the desired result. 'The subsequent 'after bathing operations' by the patient become more and more drastic until eventually a single ragged spike is left too deep in the sulcus to permit further home treatment. The broken edge now grows forward with the rest of the nail but at an angle to the main body of the plate, penetration of the dermis taking place with inflammation and possibly sepsis, with excessive granulation tissue obscuring the nail plate. At this stage the patient seeks assistance and frequently a large section of the nail plate is removed as far back as the nail matrix, or a portion of nail wall may be removed complete with granulation tissue and for six months or more the patient obtains relief from pain. If the section of nail plate has been removed as far back as the matrix with resulting damage to the 'root,' the cross-section of the new plate frequently tends to start a deviation from the normal smooth growth and the new portion of the nail is rough and quite $\stackrel{\Phi}{=}$ unlike the rest of the plate in texture. Where wedge of soft tissue has been removed from the sulcus, unusual tensions seem to be producedes around the nail wall and cause considerable pain after a few months. At this stage the patient some-c times submits to an operation for the removal of the entire nail plate, including even the distal phalanx, and is left with a scarred toe which is not only cosmetically unpleasant but very frequently远 has tiny persistent spicules of nail mixed up with $\vec{\rightarrow}$ the scar. Radical removal of the nail plate is frequently unsuccessful as the tissues of the nail $\vec{\sigma}$ matrix are particularly persistent and the patient finds considerable difficulty in trimming the spicules of nail which may push through the scar 3 tissue. Occasionally a very curious situation arises:when the distal phalanx is also removed, for the 3 . patient reports to the surgeon that the operation for the removal of the painful nail has been successful, while at the same period she is attendingo the foot clinic to see if we can help her to walk normally. One patient with a particularlyo successful surgical result was heard to remark, ' $\mathrm{I}_{N}$. have no pain but I am ashamed to expose my feeto because my toe without a nail looks revolting- $N$ almost indecent.' Fortunately this operation appears to be obsolescent, although in all fairness $\sigma$ it must be admitted that in some patients it has been successful, although in the majority the result has been unsatisfactory.

The view is widely held that where the nail plate is considerably curved, 'involuted' orō 'convoluted,' then this is an irreversible process 


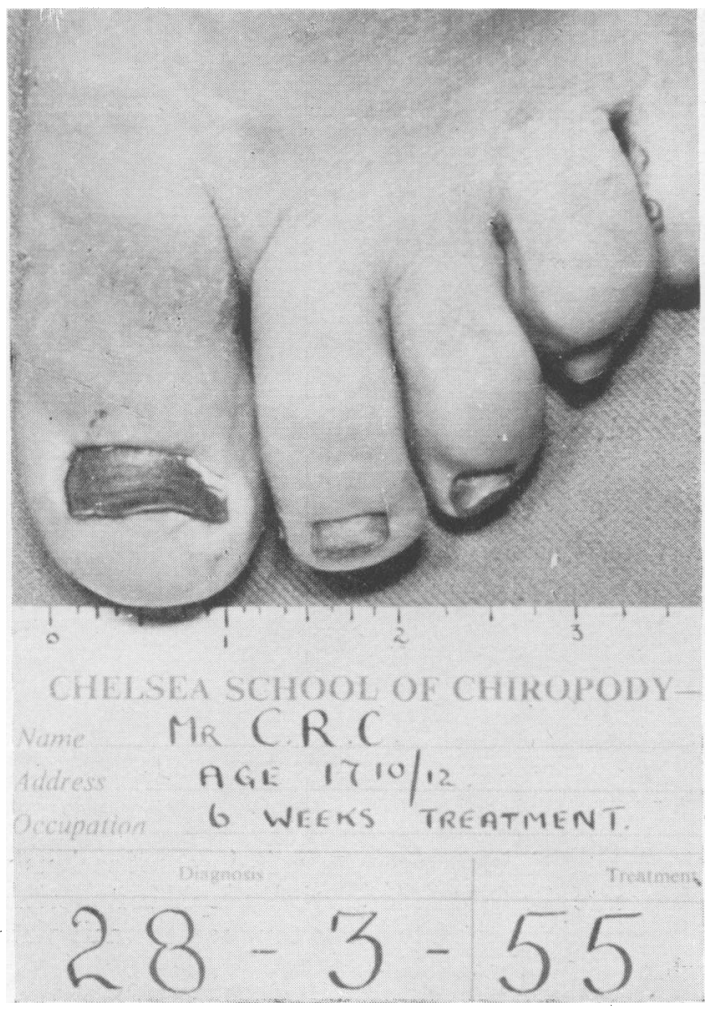

FIG. 5.-Mr. R. C. Six weeks' treatment. All granulation tissue reduced, size of toe almost normal, nail now being trained forward.

and the position can only be rectified by surgical interference with the nail. Our own experience in this direction has given very satisfactory results and shows that a highly curved nail can be straightened out and in a number of cases where the two sides have met or even overlapped at the forward edge we have been able to reduce the curvature very considerably so that the nail plate is normal.

It is perhaps necessary to explain that we, as chiropodists, try to rectify the original condition as well as give relief to the immediate painful area. This is quite important. One tends to associate ingrown nails with pointed shoes, yet one must admit that it is not the woman patient who produces the most dramatic case of ingrown nail, because there is a high incidence of ingrown nail among young men who have not been particularly athletic in their school activities and who have passed into commerce or industry, into jobs which entail long hours of standing, wearing heavy boots or tight patent leather shoes. On the other hand, however, one frequently finds that athletes, footballers and those who habitually wear short

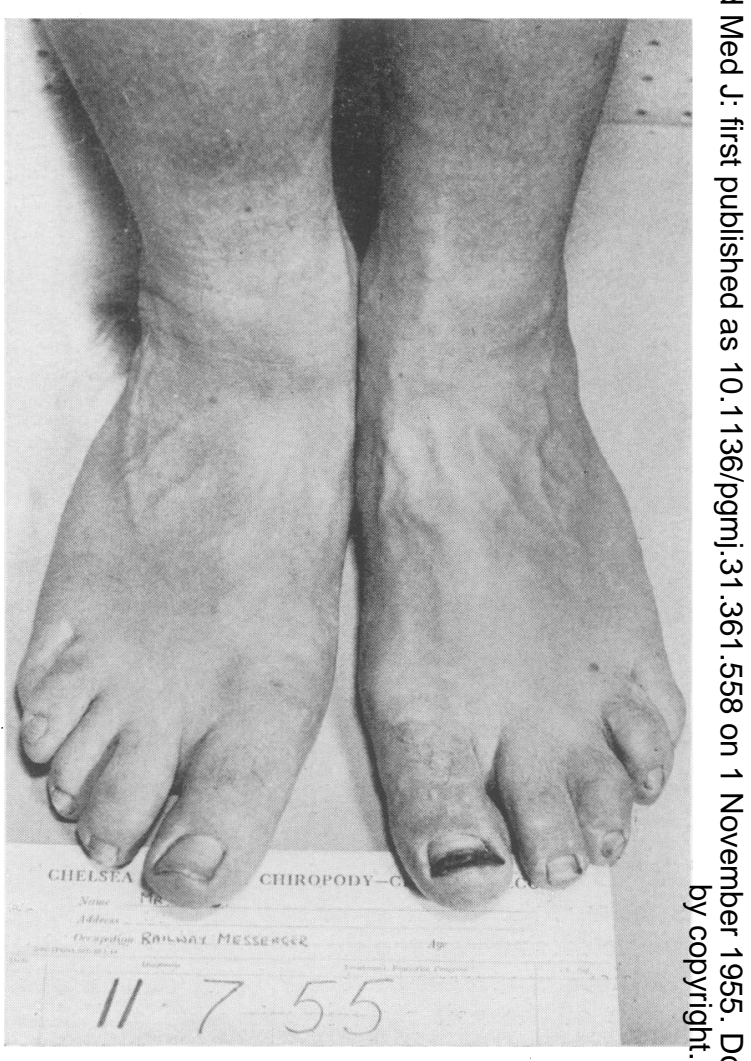

FIG. 6.-Mr. R. C. After five months' treatment. A normal nail appearing and size of toe now almost normal.

shoes for their games, produce an almost identical condition of ingrown nail. Quite often it is found that the ingrown nail subject is rather heavy, with thick fleshy toes, having an ankle which has rolled inwards, and wears a shoe which has become considerably distorted around the forepart of the inner heel counter. In these cases we $\frac{\dot{0}}{3}$ usually insert a temporary wedge to the medial border of the heel of the shoe and in some cases where the shoe sole is stout enough we insert a 'valgus' pad to' prevent this inwards collapse of 음 the foot on prolonged weight bearing. It will be $D$ appreciated that the collapse of the longitudinal arch will give unequal elongation of the inner and $N$ outer borders of the foot; this will tend to thrust os the big toe forwards and outwards and as there is $N$ seldom room for the third, fourth and fifth toes to $\underset{\omega}{N}$ move laterally in the shoe, there is a crushing of the outer border of the big toe against the second 0 toe and irritation of the lateral nail sulcus by the nail plate.

Various treatment techniques have been sug- $T$ gested, perhaps the oldest of which is to cut a 


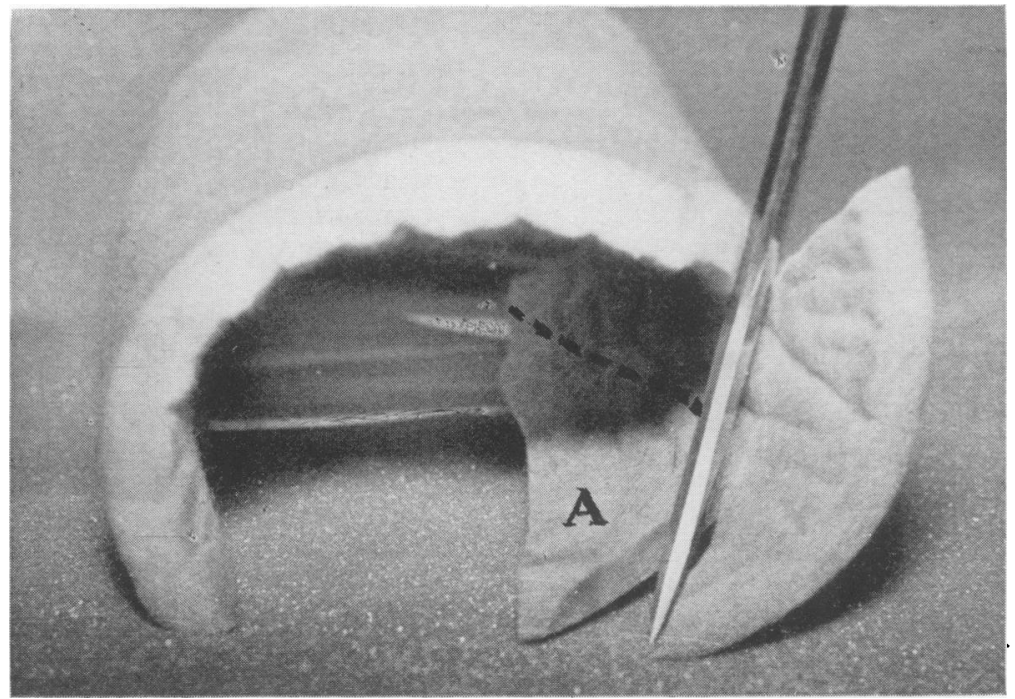

A pointed spur produced when a straight cut is made across a highly curved nail. A fine chisel is used to make the cut along the dotted line to remove the splinter ' $A$ ' before filing to produce a smooth curve.

' $\mathrm{V}$ ' in the centre of the free edge of the nail to encourage the nail to grow towards the centre and relieve pressure on the sides. This is a manifestly fallacious argument as it will be obvious that the centre of the nail plate is weakened and simply allows side pressure to increase the curvature of the nail plate still further and obviously the removal of $a^{\prime} \mathbf{V}$ ' from the centre of the nail is unlikely to reduce the total width of the hard free edge. Sometimes shallow longitudinal grooves, $\frac{1}{8}$ in. apart, are drilled in the nail from the free edge to the lunula and packing is inserted under the sides of the nail, but in many cases the packing material simply enlarges the nail groove, separates the nail plate from the nail bed and when the packing is removed the soft tissues of the nail wall tend to fall into the depression made by the packing material and there is no improvement in the condition. We use a modification of this technique which gives a fair measure of success. A strip of $\frac{1}{2}$-in. zinc oxide adhesive plaster is placed obliquely from the centre of the free edge under the nail and over the nail wall and this tends to lift the edge of the nail, depresses the nail wall to produce a flat surface and, in this case, more rapid progress can be made since packing placed between the strapping and the corner of the nail plate does not depress the soft tissues to the same degree because the strapping gives additional support.

A variety of drugs are available for the reduction of the excessive granulation tissue, among them copper sulphate and silver nitrate, but we have found that a strong solution of iron chloride gives the most satisfactory results as it does not serioysty obliterate the field, it is relatively rapid in its actrom, almost painless and tends to toughen the nail ate which in these circumstances is frequentery macerated where it is covered by granulation tişiue. If sepsis is present it is necessary to reduce it $\vec{W}$ if a suitable antiseptic and gauze drains before the iron chloride is applied and for this purpose industrial methylated spirit appears to meet moüt requirements.

Our procedure of choice is very conservative and relatively free from pain and it does give a vey high percentage of good results which appear to permanent over a period of 20 years or more.

The general technique of treatment is summarized as follows:

I. Reduce sepsis if present and remove the penetrating portion of nail. (Note that only the very minimum amount of nail consistent with the elimination of irritation should be removed.)

2. Reduce the nail thickness to normal by means of a dental burr.

3. Reduce excessive granulation tissue and with an astringent toughen the nail groove.

4. Apply $\frac{1}{2}$-in. width plaster strips diagonaPy under the front corners of the nail to suppont packing under the nail plate and, if necessary, to depress the nail wall. Keep the nail plate covered with waterproof elastoplast shaped to the nail plate.

5. After one week remove the dressings, clear the debris with ro volume hydrogen peroxide solp tion and clean with industrial spirit. With hea 
Spencer Wells or artery forceps gently crush the softened nail plate and hold in a corrected position for a few minutes. Then repeat stages 4 and 5 .

The stresses and strains thus produced at the free edge do gradually flatten out the whole of the plate back to the lunula and the tendency to produce an 'ingrown nail' is thus reduced.

No treatment is likely to be successful unless there is adequate space in the toe-box of the shoe to accommodate the toes without back pressure or side pressure, and it may be necessary to advise the patient to remove the toe-cap before continuing treatment, and in this case a moulded samcoforma ( $120 \mathrm{SB}$ ) cradle can be used as a walking splint if it is moulded directly to the toe and it is also at least as successful a protection to the toe as is the ordinary shoe used by industrial workers.

I am aware that this technique will be considered to be too time-consuming for the busy surgeon or the general practitioner, but as the results are so satisfactory in every way and as many chiropodists have both the knowledge and the skill, I consider that this is one of the conditions which might well be delegated to the chiropodist.

\section{A new book for the postgraduate}

\section{Ophthalmology}

\section{A TEXTBOOK FOR DIPLOMA STUDENTS}

\section{by P. D. TREVOR-ROPER}

M.B., B.Chir.(Camb.), F.R.C.S., D.O.M.S.(Eng.)

Curator, Dept. of Pathology, Institute of Ophthalmology;

Asst. Ophthalmic Surgeon, Westminster Hospital.

This textbook aims to cover the field of ophthalmology required by the postgraduate student seeking an ophthalmic diploma. It deals at appropriate length, and in considerable detail, with the anatomy and physiology of the eye, with physical and physiological optics including the prescription of spectacles, and then covers systematically the diseases of the outer and inner eye. The emphasis falls throughout on clinical experience.

$x v i+656 p p$. 449 illustrations 8 colour plates (1955) 75s. net

\section{A new book for the specialist}

\section{Studies on THe Cerebral Cortex}

\section{by S. RAMÓN Y CAJAL}

(Translation from the Spanish by Lisbeth M. Kraft, Yale University.)

The timeliness of this translation is self-evident from the widespread and growing interest in the limbic system. Nevertheless, a precise knowledge of anatomy is essential to a thorough elucidation of function. When Ramón y Cajal undertook and completed the series of studies included in this volume he probably gave us the most precise and comprehensive single anatomical account of the limbic system that is available.

\section{Descriptive leaflets available on request}

\section{LLOYD-LUKE (MEDICAL BOOKS) LTD., 49 Newman Street, W.1}

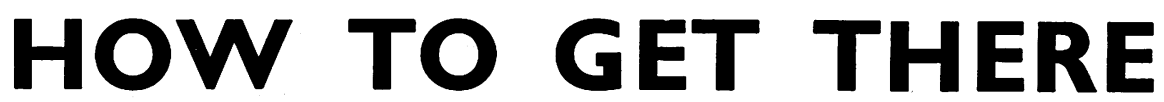

An Address Book for the Medical profession, showing how to reach the various Colleges, Societies, Institutes and Hospitals in or near London

New (Fourth) Edition: 1954

Price 2s. 6d. (2s. 9d., post free)

Published by the

FELLOWSHIP OF POSTGRADUATE MEDICINE

60 Portland Place, London, W.I

$x i i+179 p p$.

108 illustrations

(1955) 27s. 6d. net 